\title{
A mechanical lure that is highly maneuverable
}

BEATRICE T. GARDNER AND R. ALLEN GARDHER

UNIVERSITY OF NEVADA

In studies with active, unrestrained animals, the conventional fixed-track method for moving solid lures limits the extent of stimulus control. The mechanism described in this paper moves small solid objects through any pathway on a plane surface. Given such flexibility, it is possible to vary or hold constant distance, direction, and movement pattern in exposures to solid lures.

Much of the behavior of active animals consists of responses to the stimuli presented by the movements of other active animals, as, for example, the hunting behavior of predators. But precise analysis of the behavior of the responding animal frequently requires more control of the stimuli than can be obtained with live stimulus animals. Investigation of the hunting behavior of jumping spiders (Gardner, 1964, 1965, 1966) has required a mechanical system for moving three-dimensional models of prey to any position in a plane at any of a wide range of speeds. The mechanical system which we developed to accomplish this may prove useful in a variety of similar invesitgations.

In this apparatus an opaque sheet of white plastic completely covers the mechanism that moves the lures. This is the surface on which the lure is placed. Preserved specimens of actual prey, and various artificial substitutes for prey, such as small plastic hemispheres, serve as lures. The lure is glued to a very thin steel plate and is moved by its magnetic attraction to a small rider mounted directly below the opaque sheet of plastic.

This rider (see Detail-sliding Rider, Fig. 1) is pierced at right angles by two holes. Two rods are inserted through the rider by means of these holes and the rider slides freely along both rods. The rods are moved at right angles to each other by means of two independent sets of pulleys attached to the ends of the rods (Fig. 1). Thus, when the first rod (LeftRight Rod) is moved by its pulleys, the rider slides along the second rod and is carried in the left-right direction. Similarly, when the second rod (FrontBack Rod) is moved by its pulleys, the rider slides along the first rod and is carried in the front-back direction. If both rods are being moved at the same time, the rider is carried in an oblique pathway. If, in addition to pulling both rods simultaneously, the speed of movement is also varied, a variety of curved pathways can be formed. The magnetic top of the sliding rider, then, has the properties of a point in a system of Cartesian co-ordinates: it can move to any location in the plane and can describe any pathway within the plane.
Each of the two systems of pulleys is attached to a separate adjustable-speed reversible-rotation motor, and manual setting of the forward-stop-reverse switch and the variable speed dial of the control box for the motors yields the desired patterns of movement. ${ }^{2}$ Producing complex curves or circles requires considerable manual skill and, in fact, research assistants familiarize themselves with the apparatus by practicing until they can move the rider in a perfect circle. ${ }^{3}$

The testing chamber containing the spider fits over the remainder of the apparatus. The chamber is a large shallow cylinder with white opaque walls and with a clear plastic ceiling and floor. Just below the transparent floor of the spider's compartment is the surface on which the lure moves.

The apparatus is being used in experiments on the repeated elicitation of hunting in Salticid spiders. Hunting consists of a chain of responses starting with orientation at the prey (or artificial lure) and culminating in a jump that serves to capture the prey. Changes in responsiveness as a function of hunger

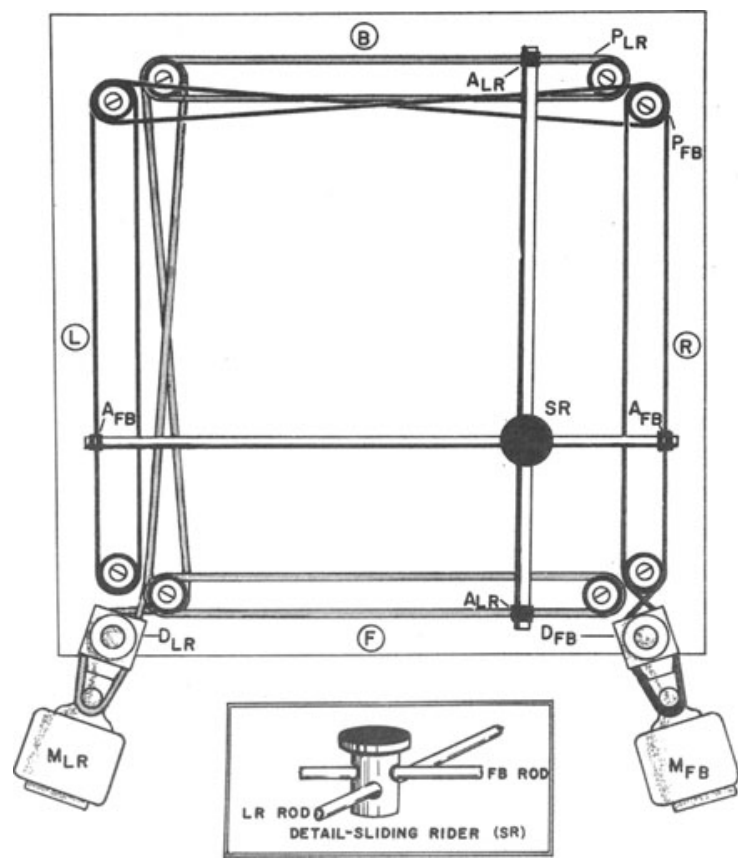

Fig. 1. A mechanical system for moving three-dimensional lures: SR-Sliding rider with magnetic top: $\mathbf{P}_{\mathbf{L R}}, \mathbf{P}_{\mathbf{F B}}-$ Left-Right and Front-Back pulley systems ( $L R$ cord shown in gray, FB cord in black); $A_{L R}, A_{F B}$-Point of attachment, Left-Right rod and FrontBack od to cond of respective pulley systems; $M_{L R}, M_{F B}-L$ eftRight and Front-Back motors; $D_{L R}, D_{F B}-$ Left-Right and FrontBack drive shaft. 
level, number of previous exposures, characteristics of the lure and its movements, and similarity of the lure in the first and second set of presentations are being investigated.

Table 1 presents an example of data obtained when the apparatus is used to produce the same pattern of movement repeatedly, a procedure especially suitable for studies on habituation of behavior to a moving target. In this particular case, the lure moved in a Z-shaped pathway under the transparent floor of a long and narrow testing chamber. Each presentation of the lure consisted of an initial slow phase (30 sec at 4 in. per $\mathrm{min}$ ) and a final fast phase (30 sec at 16 in. per min). Table 1 shows the effects of habituation and of drive on the amount of time spent orienting at the lure, both of which were significant at $p<.05$ (for deprivation, $F=4.89, d f=1 / 22$, and for successive presentations, $F=4.22, d f=2 / 44$ ).

The lure can also be moved in a response-dependent mode, thus matching the speed of the pursuing predator, or changing direction, distance, or speed after a given amount of responding, etc. This method of moving the lure is especially appropriate for specifying the stimuli for the several sequential hunting responses. Table 2 shows some of the data obtained with the response-dependent mode of movement. In this particular case, the moving lure was kept at a constant distance from the predator throughout each $30 \mathrm{sec}$ presentation. It can be seen that the characteristic response to the lure depended on its speed as well as on the hunger level of the predator. The analysis of variance shows that these conditions determine whether the spider will respond to the lure at all ( $F=3.60, d f=3 / 27, p<.05)$, and whether the spider will pursue the lure ( $F=7.49$, df $=3 / 27, p<.01$ ).

The apparatus has now worked reliably for over 10 months, throughout individual testing sessions last-

Table 1. The same pattern of movement is repeated Median amount of time spent oriented during successive $1 \mathrm{~min}$ presentations of a moving lure to the jumping spider Phidippus coccineus at two levels of food deprivation ( $N=24)$.

\begin{tabular}{cccc}
\hline \multicolumn{4}{c}{ Successive Presentations } \\
\hline Days & Eorly, 1-15 & Middle, 21-35 & Late, 36-50 \\
Deprived & & & \\
2 & $15.0 \%$ & $15.2 \%$ & $11.0 \%$ \\
7 & $32.8 \%$ & $23.7 \%$ & $16.6 \%$ \\
\hline
\end{tabular}

Table 2. The pattem of movement depends on Ss responses Characteristic response of the jumping spider $P$. coccineus to a moving lure that is kept 1 in. away during 15 presentations at each of two speeds and at each of two levels of food deprivation $(N=10)$,

\begin{tabular}{|c|c|c|c|c|}
\hline Speed & $4 \% / \min$ & $4 " 1 / \min$ & $16^{\prime \prime} / \mathrm{min}$ & $16 " / \min$ \\
\hline Days deprived & 2 & 7 & 2 & 7 \\
\hline No response & $16.7 \%$ & $13.4 \%$ & $2.5 \%$ & $3.2 \%$ \\
\hline Orientation alone & $56.9 \%$ & $50.8 \%$ & $52.0 \%$ & $37.6 \%$ \\
\hline \multicolumn{5}{|l|}{ Orientation and } \\
\hline Pursuit & $26.5 \%$ & $35.8 \%$ & $45.5 \%$ & $59.2 \%$ \\
\hline
\end{tabular}

ing several hours, and for the many hundreds of precisely repeated patterns of movement produced during each experiment. Although the principle of the mechanism is simple, the milling and mounting of the pulley systems required the help of a skilled machinist. Routine care and maintenance, including the periodic replacement of the nylon pulley cord, is within the capabilities of most students of animal behavior.

The apparatus was designed for specific studies with jumping spiders in which it was important that exposure to the moving lure be constant over repeated presentations even though the active predator might be at any place in the testing chamber, and facing in any direction. A variety of other workers in animal behavior must have faced this problem: those who wish to compare the releasing characteristics of several moving lures, those interested in the range or accuracy of tracking responses to moving targets, and those concerned with changes in responsiveness to lures as a function of repeated presentation, drive state, or age.

\section{References}

Gardner, B. T. Hunger and sequential responses in the hunting behavior of Salticid spiders. J. comp. physiol. Psychol., 1964. $58,167-173$.

Gardner, B. T. Observations on three species of Phidippus jumping spiders (Araneae: Salticidae). Psyche, 1965, 72, 133-147.

Gardner, B. T. Hunger and characteristics of the prey in the hunting behavior of Salticid spiders. J. comp. physiol. Psychol., $1966,62,475-478$.

\section{Notes}

1. Supported in part by NIH grant MH-08938.

2. The adjustable-speed reversible-rotation motors used in the present apparatus are Bodine type NSH-12R, Bodine Electric Co., Chicago, Ill. Control boxes for these motors are manufactured by Minarik Electric Co., Los Angeles, Cal.

3. Same principle as the toy Etch-a-Sketch (Ohio Art Co., Bryan, Ohio). 